\title{
Nuclear export and mitochondrial and endoplasmic reticulum localization of IGF-binding protein 3 regulate its apoptotic properties
}

\author{
Vladislava Paharkova-Vatchkova and Kuk-Wha Lee
}

Division of Pediatric Endocrinology, David Geffen School of Medicine at UCLA, Mattel Children's Hospital, 10833 Le Conte Avenue, MDCC 22-315, Los Angeles, California 90095, USA

(Correspondence should be addressed to K-W Lee; Email: kukwhalee@ mednet.ucla.edu)

\begin{abstract}
Tumor suppression by IGF-binding protein 3 (IGFBP3) may occur in an IGF-independent manner, in addition to its role as a regulator of IGF bioavailability. After secretion, IGFBP3 is internalized, rapidly localized to the nucleus, and is later detected in the cytoplasm. We identified a putative nuclear export sequence (NES) in IGFBP3 between amino acids 217 and 228, analogous to the leucine-rich NES sequence of p53 and HIV Rev. Mutation of the NES prevents nucleocytoplasmic shuttling of IGFBP3 and blocks its ability to induce apoptosis. Targeting of IGFBP3 to the mitochondria and endoplasmic reticulum (ER) was confirmed by co-localization with organelle markers using fluorescence confocal microscopy and subcellular fractionation. Mitochondrial targeting was also demonstrated in vivo in IGFBP3-treated prostate cancer xenografts. These results show that IGFBP3 shuttles from the nucleus to the mitochondria and ER, and that nuclear export is essential for its effects on prostate cancer apoptosis.
\end{abstract}

Endocrine-Related Cancer (2010) 17 293-302

\section{Introduction}

Insulin-like growth factor-binding protein 3 (IGFBP3) exerts its tumor suppressive properties via IGFdependent and -independent mechanisms (for review see Firth \& Baxter (2002)). IGFBP3 has been historically described as a modulator of cell growth through its IGF-dependent effects, specifically the transport of IGF1 to its receptor resulting in potentiation of IGF1 action, or sequestration of IGF1 from its cognate receptor, inhibiting its activity. Sequestering IGFs from signaling via their cognate receptor is an important property of IGFBP3 that could be explored in the therapy of cancer. However, IGFBP3 is now widely accepted to have additional IGF-independent properties (including apoptosis induction) that identify it as a unique peptide for cancer therapy.

Several advances in the field have enabled researchers to demonstrate evidence for IGF-independent effects of IGFBP3 using various strategies: 1) IGFBP3 mutants that do not bind to IGF1 (Buckway et al. 2001, Hong et al. 2002, Yan et al. 2004) or IGF1 analogs with reduced affinity for IGFBP3 (long R3-IGF1, GroPep, Australia, and des-(1-3)-IGF1; Yamamoto \& Murphy 1994, 1995); 2) biologically active IGFBP3 fragments with total and partial loss of IGF affinity respectively (Lalou et al. 1996); 3) transfection of an IGFBP3 overexpression vector into cells lacking the type 1 IGF receptor (Valentinis et al. 1995, Rajah et al. 1997); and 4) use of IGF1 negative cell lines (breast cancer cells and chondrocytes; Gucev et al. 1996, Spagnoli et al. 2002).

Transgenic mice overexpressing either IGFBP3 or the non-IGF-binding mutant GGG-IGFBP3 mated with a mouse model of prostate cancer demonstrated convincingly that a) IGFBP3 potently inhibits prostate cancer growth and progression, and that $b$ ) these effects are mediated by both IGF-dependent and IGF-independent mechanisms (Silha et al. 2006). Together, in vitro and in vivo studies provide convincing evidence supporting IGF-independent actions of IGFBP3.

After secretion, IGFBP3 is internalized via endocytic mechanisms and localizes to the nucleus 
(Schedlich et al. 1998, 2000, Lee et al. 2004). This transport is mediated by importin- $\beta$ and a C-terminal nuclear localization sequence (NLS; Schedlich et al. 2000). In addition, phosphorylation of IGFBP3 by DNA-dependent protein kinase enhances nuclear accumulation and its apoptotic activities (Cobb et al. 2006).

We previously proposed a role for IGFBP3 in the nucleo-mitochondrial translocation of the nuclear receptors $\mathrm{RXR} \alpha$ and Nur77 to initiate the intrinsic apoptotic pathway and caspase activation, as well as demonstrating that IGFBP3 and Nur77 associate in the cytoplasmic compartment of 22RV1 cells (Lee et al. 2005, 2007). Importantly, the nuclear transcription factors p53, Nur77, and RXR $\alpha$ have been shown to be actively transported out of the nucleus to mitochondria dependent on leucine-rich nuclear export sequences (NES; Stommel et al. 1999, Cao et al. 2004, Moll et al. 2006).

For this study, we identified sequence elements in IGFBP3 that are required for nuclear export and mitochondrial localization. We found that nuclear export is required for IGFBP3-induced apoptosis, and that exogenously administered IGFBP3 localizes to mitochondria in $22 \mathrm{RV} 1$ prostate cancer xenografts. These findings suggest that IGFBP3 may be regulated by trafficking between nuclear and extranuclear targets, and that these extranuclear targets mediate its apoptotic effects in prostate cancer cells.

\section{Materials and methods}

\section{Materials}

Insmed (Glen Allen, VA, USA) provided recombinant human IGFBP3. Commercial antibodies included anti-human IGFBP3 from DSL (Webster, TX, USA) and R\&D systems (Minneapolis, MN, USA); anti-human PDI (for ER identification) was from Stressgen (Ann Arbor, MI, USA); anti-human ACSL4 (for MAM identification) and anti-human $\mathrm{RXR} \alpha$ was from Santa Cruz Biotechnologies (Santa Cruz, CA, USA); and control goat IgG from Santa Cruz Biotechnologies. Anti-human Bcl-2 and Hsp60 were from Abcam (Cambridge, MA, USA). Nur77 antibody was in-house generated and previously described (Lee et al. 2005). DNA PKcs antibody was from Kamiya Biochemical Company (Seattle, WA, USA). SDS-PAGE reagents, Tween, and fatfree milk were purchased from Bio-Rad. ECL reagents were from Amersham. All other chemicals were from Sigma-Aldrich.

\section{Cell culture}

22RV1 cells were maintained in RPMI 1840 medium containing 10\% FCS (Life Technologies), 100 units of penicillin/ml, and 100 units of streptomycin/ml in a humidified environment with $5 \% \mathrm{CO}_{2}$. Fibroblasts from an IGF1 receptor knockout and corresponding wild-type (WT) mouse were a kind gift from Dr Renato Baserga (Sell et al. 1993, Valentinis et al. 1995). These cells are designated receptor-negative $(\mathrm{R}-)$ embryonic fibroblast cells (MEFs). The R-cells were maintained in DMEM containing 10\% fetal bovine serum and geneticin (G418). All cells were used before passage 6 .

\section{Site-directed mutagenesis}

Mutant NES IGFBP3 protein was constructed by conversion of two leucine residues - at positions 224 and 227 to alanines by second-round PCR overlap mutagenesis. Stratagene's QuikChange Primer Design Program at www.stratagene.com/qcprimerdesign was used for designing primers to introduce the site-directed mutations. Primers GGAAGACACACTGAATCACGCGAAGTTCGCCAATGTGCTGAGTCCCAGG and CCTGGGACTCAGCACATTGGCGA ACTTCGCGTGATTCAGTGTGTCTTCC were used to generate the ${ }^{224} \mathrm{~L}$ to $\mathrm{A}$ and ${ }^{227} \mathrm{~L}$ to A double mutant. Underlined are the nucleotides introducing the mutations.

For subcloning into the pFLAG CMV vector (Sigma) and C-terminal FLAG sequence, fusion mutants were amplified with engineered EcoRI and KpnI restriction sites to the sense and antisense primers respectively: GCTGAATTCCCACCATGCAGCGGGCGCGACCCACG and ATCGGTACCCTACTTGTCATCGTCGTCCTTGTAATCCTTGCTCTGCATGCTGTAGCAGTGC. Mutants were confirmed by sequencing.

\section{Transient transfections and cell death ELISA}

Cells $\left(2 \times 10^{4}\right)$ were seeded in 96-well culture plates. Reagents were appropriately scaled up to six-well plates for transfections that were followed by mitochondrial isolation and subsequent western immunoblotting. Transfections were done with LipofectAMINE:PLUS Reagent as directed by the manufacturer (Invitrogen). Typically, $50 \mathrm{ng}$ $\beta$-galactosidase expression vector (pSV- $\beta$-Gal, Promega) and $50 \mathrm{ng}$ expression vector were mixed with carrier DNA to give $0.2 \mu \mathrm{g}$ total DNA per well. After $24-48 \mathrm{~h}$ of transfection, photometric Cell Death ELISA (Roche Applied Science) was 
performed according to the manufacturer's instructions to quantify histone-associated DNA fragments (mono- and oligonucleosomes) generated by apoptotic cells.

\section{Co-immunoprecipitation and western immunoblots}

22RV1 nuclear or cytoplasmic extracts were immunoprecipitated with control IgG, anti-IGFBP3, or anti$\mathrm{RXR} \alpha$ antibodies. Briefly, $250 \mu \mathrm{l}$ of protein A-agarose were incubated overnight at $4{ }^{\circ} \mathrm{C}$ with $5 \mu \mathrm{l}$ of antihuman IGFBP3 antibodies. About $125 \mu \mathrm{l}$ of each antibody-treated protein A-agarose were added to $10 \mu \mathrm{g}$ protein extract and incubated for $3 \mathrm{~h}$ at $4{ }^{\circ} \mathrm{C}$ with shaking. Immunoprecipitated proteins were pelleted by centrifugation and washed three times with $500 \mu \mathrm{l}$ IP wash buffer $\left(1 \times \operatorname{PBS}\left(\mathrm{Ca}^{2+} \mathrm{Mg}^{2+}\right.\right.$ free $)$, $0.05 \% \mathrm{NP} 40,0.02 \% \mathrm{NaN}_{3}$ ). Two hundred microliters sample buffer was added to each sample and vortexed vigorously. Samples were boiled and vortexed again to release protein-antibody complexes from the protein A-agarose. The protein A-agarose was then separated from the immunoprecipitated complexes by centrifugation. The supernatants were saved, and the immunoprecipitated proteins were separated by nonreducing SDS-PAGE (8\%) at constant voltage overnight, then transferred to PVDF for $4 \mathrm{~h}$ at $170 \mathrm{~mA}$. The nitrocellulose was immersed in blocking solution (5\% nonfat milk/PBS) for $45 \mathrm{~min}$, washed with TST wash buffer $(0.15 \mathrm{M} \mathrm{NaCl}, 0.01 \mathrm{M}$ Tris base, $0.3 \%$ Tween 20, pH 7.4), 0.1\% Tween, and incubated with primary anti-human IGFBP3 antibody (1:4000) for $2 \mathrm{~h}$. After washing off any unbound antibodies, the PVDF was incubated with a secondary antibody (1:10 000) for $1 \mathrm{~h}$. The membrane was washed four times with TST wash buffer. Bands were visualized using the peroxidaselinked enhanced chemiluminescence detection system (ECL, Amersham Pharmacia Biotech). Experiments were repeated three times.

\section{Subcellular fractionation procedures}

$\mathrm{Nu}$-CLEAR Protein Extraction Kit was from SigmaAldrich, and isolation was followed according to the manufacturer's protocol.

\section{Isolation of mitochondria, endoplasmic reticulum, and mitochondria associated membrane fractions}

Membrane-associated fractions were isolated according to the published protocols (Bozidis et al. 2007). This procedure combines differential centrifugation (for separation of the postnuclear supernatant from nuclei and cellular debris at low $\boldsymbol{g}$ forces) and a selfgenerating Percoll gradient centrifugation.

\section{Immunoflourescence confocal microscopy}

For overexpression staining, 30000 cells per chamber were plated, and $200 \mathrm{ng}$ plasmid was transfected using Lipofectamine 2000 (Invitrogen) and cells were used for immunohistochemistry $48 \mathrm{~h}$ after transfection. Cells were stained live with MitoTracker Deep Red 633 FM (Molecular Probes, Carlsbad, CA, USA) according to the manufacturer's instructions. Cells were fixed for $10 \mathrm{~min}$ in $4 \%$ paraformaldehyde in PBS and permeabilized in $0.2 \%$ Tween 20. Incubations with primary antibody and secondary reagent were done for $1 \mathrm{~h}$ at room temperature. For the FLAG-tagged IGFBP3 construct, anti-FLAG antibody rabbit polyclonal (Cell Signaling, Danvers, MA, USA) was used at $400 \times$ dilution, followed by Alexa Fluor 488 Goat Anti-Rabbit conjugate (Molecular Probes) at 1:400, i.e. $5 \mu \mathrm{g} / \mathrm{ml}$ final concentration. Counterstaining of nuclei was done with DAPI, and cells were mounted in ProLong Gold antifade reagent (Molecular Probes). Acquisitions were done using Leica DM IRE2 Microscope and Leica Confocal Software. Endogenous IGFBP3 was detected with anti-human IGFBP3 goat polyclonal antibody (R\&D), followed by Alexa Fluor 488 (Molecular Probes). Endoplasmic reticulum (ER) was labeled with anti-human PDI mouse monoclonal antibody (Stressgen), followed by Alexa Fluor 633 rabbit anti-mouse IgG (Molecular Probes). For ER staining, samples were stained according to the manufacturer's references (i.e. only methanol fixation/permeabilization for $20 \mathrm{~min}$ at $-20^{\circ} \mathrm{C}$ was carried without fixation in paraformaldehyde).

\section{Tumor xenografts}

22RV1 xenograft tumors were generated by injection of $1 \times 10^{6}$ cells in $200 \mu 1$ mixed at a 1:1 dilution with Matrigel in the right flank of male SCID mice. Tumors were established for 2 weeks before the start of treatment. Ten SCID mice with 22RV1 tumors were treated twice per day with saline or IGFBP3 $(40 \mathrm{mg} / \mathrm{kg}$ per day) given by divided i.p. injections. The mice were killed after 3 days. Tumors were harvested, and immediately processed with Mitochondria Isolation Kit (animal tissue specific, Sigma). Mitochondrial proteins were resolved by SDS-PAGE and immunoblotted with IGFBP3 antibody (DSL). 


\section{Statistical analysis}

All experiments were repeated at least three times. Means \pm s.D. are shown. Statistical analyses were performed using ANOVA utilizing InStat (GraphPad, San Diego, CA, USA). Differences were considered statistically significant when $P<0.005$, denoted by **.

\section{Results}

\section{Rapid IGF-independent nuclear localization of IGFBP3 and cytoplasmic export}

Nuclear localization of IGFBP3 is a well-described phenomenon and has been demonstrated in a variety of cellular models (Jaques et al. 1997, Li et al. 1997, Wraight et al. 1998, Liu et al. 2000, Sun et al. 2008). IGFBP3 possesses a consensus bipartite NLS (Radulescu 1994), and nuclear transport is facilitated by importin- $\beta$ factor (Schedlich et al. 2000). We have begun to characterize the intracellular trafficking of IGFBP3 and its relation to biological function. We have also recently published mechanisms involved in the internalization of IGFBP3 after secretion (Lee et al. 2004). After internalization by endocytic pathways, IGFBP3 is first targeted to the nucleus (Fig. 1A). About $500 \mathrm{ng}$ of rhIGFBP3 was added to $22 \mathrm{RV} 1 \mathrm{CaP}$ cells for $15 \mathrm{~min}$. Subcellular fractions were isolated, and IGFBP3 was immunoprecipitated with goat polyclonal anti-IGFBP3 antibody. Immunoprecipitation of treated cell lysate with control IgG is also shown on the right. Proteins were resolved by SDS-PAGE, and IGFBP3 was identified by immunoblotting with mouse monoclonal anti-IGFBP3 antibody. Within $15 \mathrm{~min}$ of addition of IGFBP3, we were able to detect IGFBP3 in the nucleus, suggesting that this is the initial destination of internalized IGFBP3. To expand the

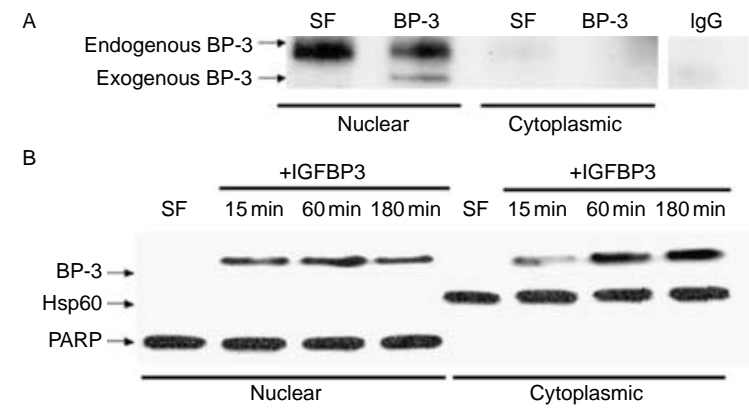

Figure 1 IGFBP3 enters the nucleus rapidly and subsequently is exported to the cytoplasm. (A) 22RV1 CaP cells treated with IGFBP3 for $15 \mathrm{~min}$, and subcellular fractions were immunoprecipitated and immunoblotted with IGFBP3 antibody. (B) Time course of IGFBP3 subcellular localization in R-MEF cells, Hsp60, and PARP were used to assess purity of the subcellular fractions. SF, serum-free control. time course and explore the IGF-independent intracellular trafficking of IGFBP3, we utilized IGF $\mathrm{R}-$ MEFs derived from an IGF1R knockout mouse. These cells have been shown previously to neither bind nor respond to IGFs (Sell et al. 1993). R-MEFs were pulsed with $500 \mathrm{ng}$ of IGFBP3, and its subcellular localization was followed over a $180 \mathrm{~min}$ time course (Fig. 1B). To demonstrate the purity of the fractionation, expression of mitochondria-specific protein Hsp60 and nuclear-specific protein poly(ADP-ribosyl) polymerase (PARP) is shown. Again, the primary destination of IGFBP3 in $15 \mathrm{~min}$ is the nucleus, after which levels in the cytoplasm begin to increase, consistent with an active export mechanism.

\section{A highly conserved NES lies within the C-terminal domain of IGFBP3}

Because of the preliminary data in Fig. 1 showing the shuttling of IGFBP3 from the nucleus to the cytoplasm, we examined the primary amino acid sequence of IGFBP3 to determine whether it contains a leucine-rich sequence of conserved spacing and hydrophobicity, which fits the criteria established for an NES (Bogerd et al. 1996, Kim et al. 1996). We observed that the C-terminal residues between amino acids 190 and 201 conform to this motif, as indicated by their similarity to other known NESs such as HIV Rev and p53 (Table 1; Fischer et al. 1995, Stommel et al. 1999). In addition, alignment of this sequence across other members of the IGFBP family is shown. This sequence is highly conserved in widely divergent species. In addition to its role as a nuclear transcription factor, p53 has a confirmed pro-apoptotic role at the mitochondria (Mihara et al. 2003). This putative sequence suggests extranuclear trafficking of IGFBP3 and the possibility of a mitochondrial function for IGFBP3.

Table 1 A nuclear export sequence (NES) in insulin-like growth factor-binding protein 3 (IGFBP3)

\begin{tabular}{lrlr}
\hline p53 & 340 & MFRELNEALELK & 351 \\
HIV rev & 72 & LP-PL-ERLTLD & 84 \\
BP-3 & 217 & MEDTL-NHLKFL & 227 \\
BP-5 & 196 & MEASL-QELKAS & 206 \\
BP-4 & 178 & LHRAL-ERLAAS & 188 \\
BP-2 & 234 & LDQVL-ERISTM & 244 \\
BP-6 & 167 & LDSVL-QQLQTE & 177 \\
BP-1 & 194 & SGEEI-SKFYLP & 204 \\
\hline
\end{tabular}

This region was identified in the C-terminal region of IGFBP3 between AA 217 and 228 due to similarity to established NES in regions of conserved spacing and hydrophobicity in p53 and HIV Rev proteins. Analogous regions of other members of the IGFBP family are shown in descending order of similarity. Hydrophobic amino acids are highlighted in bold. 
We constructed a mutant NES IGFBP3:FLAG (C-terminal) fusion consisting of leucine to alanine conversions at residues 197 and 200, since analogous mutations in other NES-containing proteins have been reported to prevent nuclear export (Bogerd et al. 1996, Kim et al. 1996, Stommel et al. 1999). WT or mutant NES:FLAG constructs were cloned in expression vectors, verified by sequencing, and transiently transfected to assess subcellular localization.

Western immunoblotting revealed that anti-IGFBP3 antibody recognized the mutant NES (Fig. 2A). Fractionation of transfected 22RV1 prostate cancer cells into nuclear and cytoplasmic fractions revealed that whereas WT IGFBP3 had equal distribution between nuclear and cytoplasmic fractions $48 \mathrm{~h}$ after transfection, IGFBP3 NES mutant displayed increased expression in nuclear versus cytoplasmic fractions compared with WT IGFBP3 (Fig. 2B). Densitometric quantitation of the IGFBP3 bands revealed a dramatic increase in nuclear retention of IGFBP3 with concomitant decrease in cytoplasmic localization. Immunoblotting with DNA PKcs and with Hsp60 were used to assess purity of the nuclear and cytoplasmic fractions respectively.

Analysis by indirect immunofluorescent confocal microscopy correlated with subcellular fractionation for IGFBP3 localization with the mutant NES, showing increased accumulation in the nucleus (Fig. 2C).

\section{Impaired nuclear export of IGFBP3 retains RXR $\alpha /$ Nur77 heterodimers in the nucleus and abolishes the apoptotic actions of IGFBP3}

We have previously described nucleo-mitochondrial translocation of nuclear receptor RXR $\alpha /$ Nur77 heterodimers to activate the mitochondrial pathway in apoptosis induction by IGFBP3 (Lee et al. 2005, 2007). We studied the effect of overexpressing mNES IGFBP3 on the subcellular localization of RXR $\alpha /$ Nur77. Mutation of the IGFBP3 NES did not impair the interaction with RXR $\alpha$ or Nur77 (data not shown). mNES as well as WT IGFBP3 expression vector was transiently transfected in $22 \mathrm{RV} 1 \mathrm{CaP}$ cells, and cell lysates were fractionated into nuclear and cytoplasmic fractions. Fractions were then immunoprecipitated with anti-RXR $\alpha$ antibody and immunoblotted with anti-Nur77. Overexpression of mNES IGFBP3 results in a marked increase in nuclear retention of RXR $\alpha /$ Nur77 (Fig. 3A), impairing nuclear export of important mediators of the apoptotic action of IGFBP3.

To directly examine the functional relationship between the localization of IGFBP3 and its effects on apoptosis, we transiently transfected $22 \mathrm{RV} 1$ prostate cancer cells with expression vector, WT IGFBP3, and mNES IGFBP3 (Fig. 3B). Transfection of WT IGFBP3 in 22RV1 cells increased oligonucleosomal fragments, as measured by a cell death ELISA, by $56 \%$ compared with empty vector transfection control. Retaining IGFBP3 in the nucleus by mutating the NES completely inhibited the ability of IGFBP 3 to induce apoptosis. These studies confirm the integral role of extranuclear trafficking of IGFBP3 via its NES for its apoptotic properties.

\section{Association of IGFBP3 with mitochondria and ER in vitro}

In an attempt to obtain more detailed information on IGFBP3 subcellular protein localization of endogenous IGFBP3 in 22RV1 prostate cancer cells, we utilized a subcellular fractionation that combines differential and Percoll gradient centrifugations, as this is the preferred method for higher purity fractions (Bozidis et al. 2007). The internal membranes were segregated into mitochondria (Fig. 4A, lane 1), mitochondria-associated membrane (MAM; lane 2), ER (lane 3), and a pellet from the MAM fraction that was collected at low $g$ centrifugation, representing an intermediate zone between mitochondria and MAM fraction (lane 4). The MAM fraction, a subdomain of the ER which consists of membrane tubules that provide direct physical contact between the ER and mitochondria, was fractionated to high purity (Vance 1990). The presence of IGFBP3 in the various membrane fractions was assessed by immunoblotting. The relative purity of the fractions was assessed by the presence of specific marker antibodies (Hsp60-mitochondria; PDI-ER; ACSL4-MAM). Under baseline conditions in vitro, IGFBP3 localizes to the mitochondria (lane 1), and is even more abundantly represented in the ER and MAM membrane fractions (lanes 2 and 3 respectively). Bcl-2 expression is detected in all membrane fractions, as previously described (Lithgow et al. 1994). RXR $\alpha$ was detected most prominently in the mitochondria fraction, as well as a faint presence in the MAM. The presence of $\mathrm{RXR} \alpha$ in lane 4 may represent mitochondria in this transitional layer. In addition, Nur77, another binding partner of IGFBP3, was identified in the MAM and ER, with faint presence in the mitochondrial fraction. Mitochondrial localization of $\mathrm{RXR} \alpha$ and Nur77 has been described previously (Cao et al. 2004). Immunoflourescence studies revealed co-localization of endogenous IGFBP3 with Mitotracker and PDI, an ER marker, consistent with the subcellular fractionation studies (Fig. 4B). 

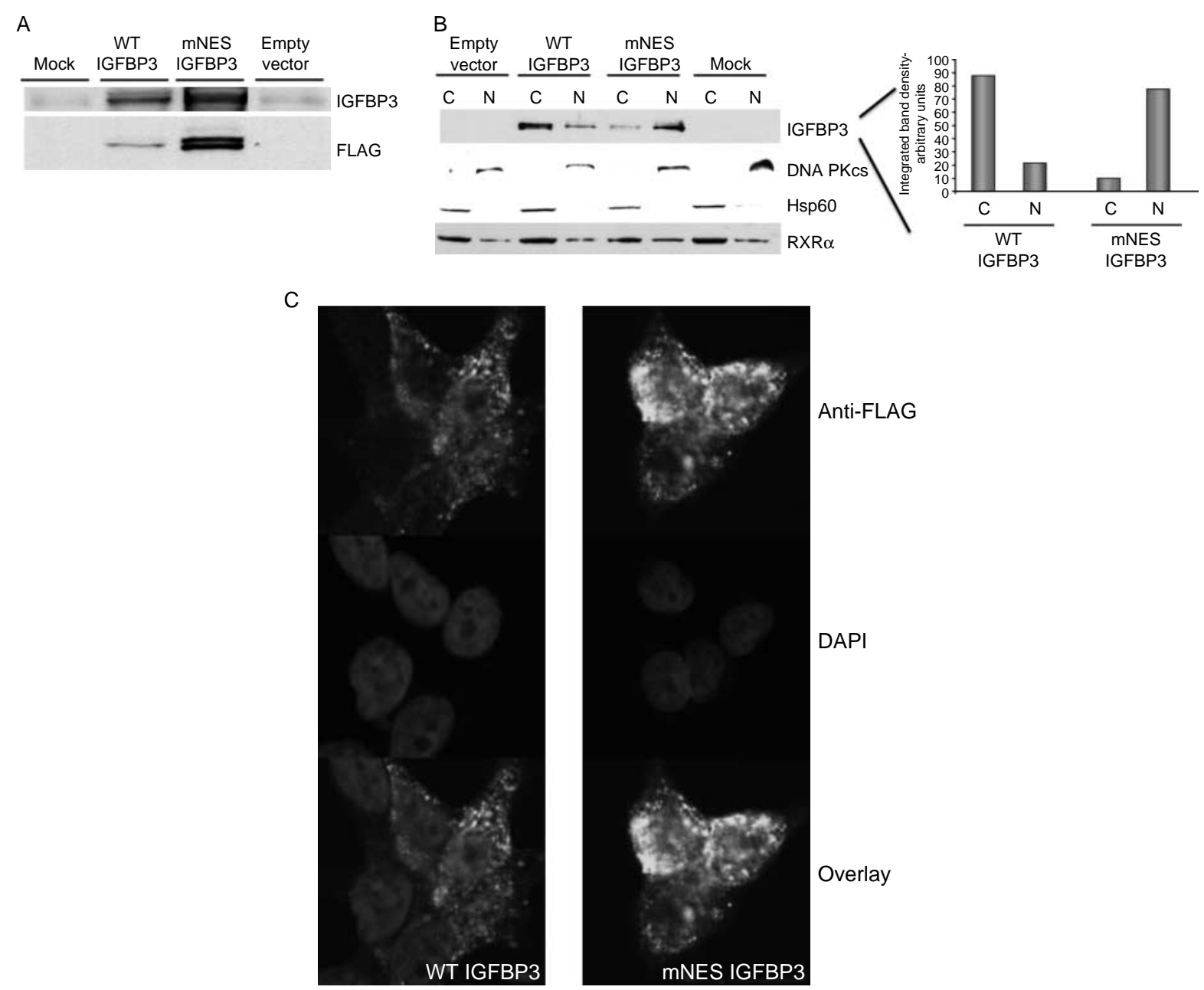

Figure 2 Subcellular localization of IGFBP3 mutants. (A) Recognition of mutant IGFBP3 by IGFBP3 antibody. (B) mNES IGFBP3 accumulates in the nucleus. 22RV1 cells were transfected with WT and mutant IGFBP3 expression vectors, and fractionated into cytoplasmic and nuclear fractions $48 \mathrm{~h}$ after transfection. IGFBP3 NES mutant shows increased amounts in nuclear versus cytoplasmic fractions compared to WT IGFBP3. Quantitation of densitometry for IGFBP3 shown on right. C, cytoplasmic fraction; $\mathrm{N}$, nuclear fraction. (C) Immunofluouresence of transfected WT and mutant IGFBP3 in $22 \mathrm{RV} 1$ prostate cancer cells. Green, anti-FLAG (IGFBP3); blue, DAPI (nuclear staining).

\section{Mitochondrial targeting of IGFBP3 in vivo}

To determine whether administered IGFBP3 is targeted to mitochondria in vivo, we administered saline or recombinant human IGFBP3 (Insmed, Inc.) at $40 \mathrm{mg} / \mathrm{kg}$ i.p. divided twice a day for 3 days to male SCID mice carrying 22RV1 prostate cancer tumors. Over this short-time period, tumor size did not decrease (data not shown) as we have described for a longer incubation (Liu et al. 2007), and as others have described in a transgenic model utilizing a non-IGFbinding mutant (Cohen 2006, Silha et al. 2006). Mitochondria were isolated utilizing the Sigma animal tissue mitochondrial isolation kit, and associated proteins were resolved by SDS-PAGE (Fig. 5).
In three of four tumors examined, IGFBP3 was found to be associated with mitochondria. Hsp60 and PARP were used to assess purity of the mitochondrial fraction.

\section{Discussion}

In the present study, we have expanded on previous structural elements that regulate the intracellular localization of IGFBP3, namely a C-terminal NLS (Schedlich et al. 2000). In the nucleus, IGFBP3 may interact with the nuclear receptors $\mathrm{RXR} \alpha$ and RAR to modulate DNA transcription (Liu et al. 2000, Schedlich et al. 2004), not unlike related binding protein IGFBP5 which also localizes to cell nuclei and 


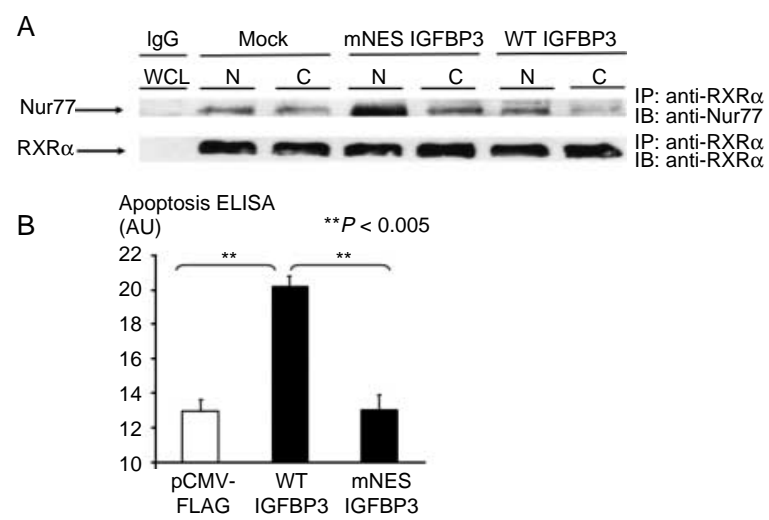

Figure 3 Nuclear export is required for the apoptotic actions of IGFBP3. (A) Overexpression of mNES IGFBP3 in 22RV1 cells induces nuclear retention of RXR $\alpha /$ Nur77 protein complexes compared to WT IGFBP3 overexpression. Mock transfection and co-immunoprecipitation with control IgG are shown on the left. After overexpression, cell lysates were fractionated and immunoprecipitation was performed as indicated. (B) Transfection of wild-type IGFBP3 in 22RV1 cells increased oligonucleosomal fragments by $56 \%$ compared to empty vector transfection control. Retaining IGFBP3 in the nucleus by mutating the NES blocked the ability of IGFBP3 to induce apoptosis.

interacts with $\mathrm{RXR} \alpha, \mathrm{RAR}$, and the vitamin $\mathrm{D}$ receptor (Schedlich et al. 2004, 2007). In addition, nuclear ubiquitination of IGFBP3 on lysine residues with subsequent degradation has been described (Santer et al. 2006). We have demonstrated cytoplasmic interaction of endogenous IGFBP3 with the nuclear receptor Nur77 (Lee et al. 2007). Upon addition of exogenous IGFBP3, RXR $\alpha /$ Nur77 heterodimers translocate to mitochondria and activate the intrinsic apoptotic pathway (Lee et al. 2005).
Upon internalization by the cell, IGFBP3 is rapidly endocytosed to the nucleus and is actively transported to the cytoplasm, since mutations in the NES cause nuclear accumulation of IGFBP3. We also describe the mitochondrial localization of endogenous IGFBP3, as evidenced by subcellular fractionation and immunofluouresence confocal microscopy. Significantly, we have recently published that IGFBP3 and BAX interaction at the mitochondria activates testicular germ cell apoptosis (Jia et al. 2009). IGFBP1 has been reported to be up-regulated in response to p53 activation and antagonizes p53 mitochondrial accumulation secondary to BAK inactivation at mitochondria in hepatocytes (Leu \& George 2007). However, IGFBP1 has not been described in the nucleus and is most dissimilar to the other IGFBPs in the leucine-rich hydrophobicity characteristic of NES (Table 1). This, combined with the fact that sequence analysis by subcellular localization bioinformatics programs does not indicate a mitochondrial targeting sequence in IGFBP1 (data not shown), means that intracellular trafficking similar to what we describe for IGFBP3 has not been previously demonstrated.

We also describe the initial description of endogenous localization for IGFBP3 and its binding partner the nuclear receptor Nur77 in the ER. Although we cannot rule out that IGFBP3 presence in the ER is not evidence of de novo ribosomal synthesis, complete absence of other probed proteins (e.g. $\operatorname{RXR} \alpha$ and Hsp60) would argue against constitutive background translation. Signaling pathways emanating from the ER are involved in apoptosis initiated by stimuli as diverse as ER stress, oncogene expression, death
A

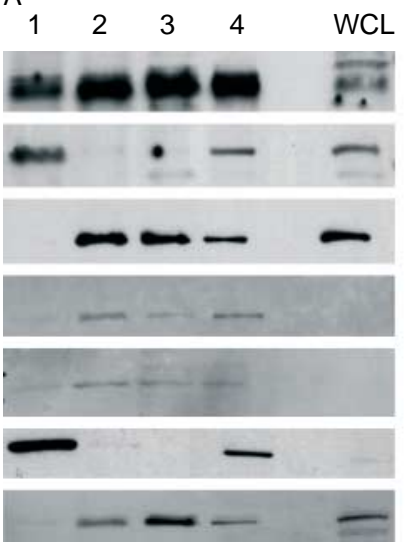

B

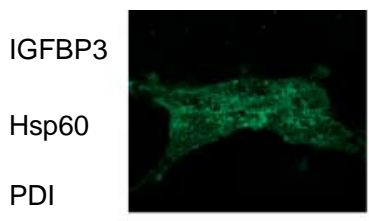

IGFBP3

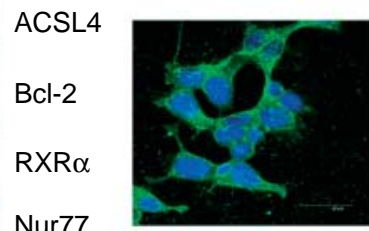

IGFBP3

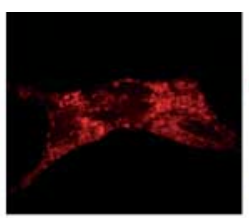

Mitotracker

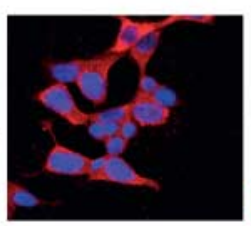

PDI

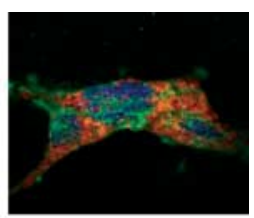

Overlay

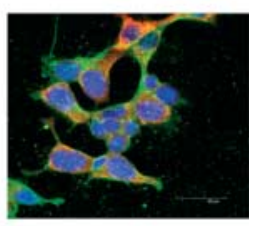

Overlay

Figure 4 Endogenous mitochondria and ER localization of IGFBP3. (A) 22RV1 prostate cancer cells were fractionated into lane 1, mitochondrial fraction; lane 2, mitochondria-associated membranes (MAM); lane 3, endoplasmic reticulum (ER); and lane 4, pellet from MAM. (b) Co-localization (yellow) of IGFBP3 (green) with mitochondrial (Mitotracker, red) and ER markers (PDI, red) by immunofluouresence confocal microscopy. 


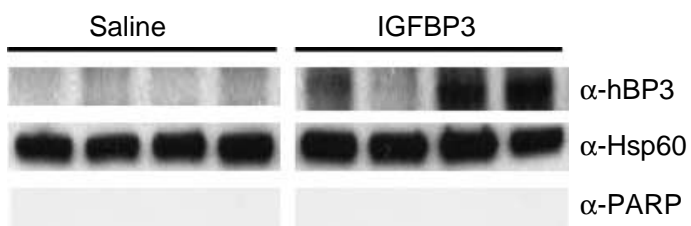

Figure 5 Prostate cancer xenografts internalize recombinant human IGFBP3 (rhIGFBP3), which localizes to mitochondria in vivo. 22RV1 CaP xenografts on SCID male mice were treated with IGFBP3, mitochondria were isolated, and immunoblotted for IGFBP3.

receptor ligation, and oxidative stress (Malhotra \& Kaufman 2007, Heath-Engel et al. 2008). Multiple pathways may be involved in ER stress-initiated apoptosis, including crosstalk with the mitochondria (Hetz 2007). IGFBP3 has been associated with a rapid increase in intracellular $\mathrm{Ca}^{2+}$ concentrations (Ricort et al. 2002). The authors proposed the activation of a G-coupled protein by IGFBP3 as this was a pertussis toxin-sensitive pathway. However, radio-iodinated IGFBP3 was utilized, and in our experience, it does not internalize into cells (data not shown). As the ER lumen represents that the major site of intracellular $\mathrm{Ca}^{2+}$ storage and release has been implicated in ER:mitochondria apoptosis cross-talk (Scorrano et al. 2003), it is intriguing to speculate that IGFBP3 ER localization may have a role in this process. The observation of IGFBP3 localization to the ER and mitochondria may in part explain previous observations that a nonsecreted mutant of IGFBP3, that did not localize to the nucleus but was primarily cytoplasmic, induced apoptosis (Bhattacharyya et al. 2006).

Functional contacts between distinct organelles have been identified and fractionated. For example, the contact sites between ER and mitochondria are being characterized as sites for exchange of calcium (Rizzuto et al. 1998) and lipids (Stone \& Vance 2000) between these organelles. In all, $\sim 5-20 \%$ of the mitochondrial network surface within a cell is in close apposition to the ER (Rizzuto et al. 1998). We have documented the presence of IGFBP3 in MAM fraction, a subdomain of the ER, which consists of membrane tubules that provide direct physical contact between the ER and mitochondria (Vance 1990). These connections, as well as the ER and mitochondria organelles themselves, are quite variable and can undergo rapid changes in overall morphology, size, and composition (Bereiter-Hahn et al. 2008). Factors such as calcium homeostasis, cell metabolism, and perceived stress can have dramatic impact on both the form and function of these organelles.
In summary, we have described extranuclear trafficking of IGFBP3 and described mitochondrial and ER localization in human prostate cancer cells. IGFBP3 is well recognized to associate with cell surface membranes (Yamanaka et al. 1999, Mishra et al. 2004), and now, we have begun to characterize its association with intracellular membrane-bound organelles. Further investigation into regulation of its subcellular localization and trafficking may provide insights into the molecular mechanisms by which it induces apoptosis and provide further rationale for translation as applied to cancer therapeutics.

\section{Declaration of interest}

The authors declare that there is no conflict of interest that could be perceived as prejudicing the impartiality of the research reported.

\section{Funding}

This work was supported in part by grants from the SteinOppenheimer Foundation, the Lawson Wilkins Pediatric Endocrinology Society, UCLA Prostate Cancer SPORE Career Development Award, National Institutes of Health Grant 2K12HD34610 (to K-W Lee), and a Department of Defense award PC061077 (to K-W Lee).

\section{References}

Bereiter-Hahn J, Voth M, Mai S \& Jendrach M 2008 Structural implications of mitochondrial dynamics. Biotechnology Journal 3 765-780.

Bhattacharyya N, Pechhold K, Shahjee H, Zappala G, Elbi C, Raaka B, Wiench M, Hong J \& Rechler MM 2006 Nonsecreted insulin-like growth factor binding protein-3 (IGFBP-3) can induce apoptosis in human prostate cancer cells by IGF-independent mechanisms without being concentrated in the nucleus. Journal of Biological Chemistry $28124588-24601$.

Bogerd HP, Fridell RA, Benson RE, Hua J \& Cullen BR 1996 Protein sequence requirements for function of the human T-cell leukemia virus type 1 Rex nuclear export signal delineated by a novel in vivo randomization-selection assay. Molecular and Cellular Biology 16 4207-4214.

Bozidis P, Williamson CD \& Colberg-Poley AM 2007 Isolation of endoplasmic reticulum, mitochondria, and mitochondria-associated membrane fractions from transfected cells and from human cytomegalovirus-infected primary fibroblasts. Current Protocols in Cell Biology Chapter 3 Unit 3.27.

Buckway CK, Wilson EM, Ahlsen M, Bang P, Oh Y \& Rosenfeld RG 2001 Mutation of three critical amino acids of the N-terminal domain of IGF-binding protein-3 essential for high affinity IGF binding. Journal of Clinical Endocrinology and Metabolism 86 4943-4950. 
Cao X, Liu W, Lin F, Li H, Kolluri SK, Lin B, Han YH, Dawson MI \& Zhang XK 2004 Retinoid X receptor regulates Nur77/TR3-dependent apoptosis (corrected) by modulating its nuclear export and mitochondrial targeting. Molecular and Cellular Biology 24 9705-9725.

Cobb LJ, Liu B, Lee KW \& Cohen P 2006 Phosphorylation by DNA-dependent protein kinase is critical for apoptosis induction by insulin-like growth factor binding protein-3. Cancer Research 66 10878-10884.

Cohen P 2006 Insulin-like growth factor binding protein-3: insulin-like growth factor independence comes of age. Endocrinology 147 2109-2111.

Firth SM \& Baxter RC 2002 Cellular actions of the insulinlike growth factor binding proteins. Endocrine Reviews $\mathbf{2 3}$ 824-854.

Fischer U, Huber J, Boelens WC, Mattaj IW \& Luhrmann R 1995 The HIV-1 rev activation domain is a nuclear export signal that accesses an export pathway used by specific cellular RNAs. Cell 82 475-483.

Gucev ZS, Oh Y, Kelley KM \& Rosenfeld RG 1996 Insulinlike growth factor binding protein 3 mediates retinoic acid- and transforming growth factor beta2-induced growth inhibition in human breast cancer cells. Cancer Research 56 1545-1550.

Heath-Engel HM, Chang NC \& Shore GC 2008 The endoplasmic reticulum in apoptosis and autophagy: role of the BCL-2 protein family. Oncogene 27 6419-6433.

Hetz CA 2007 ER stress signaling and the BCL-2 family of proteins: from adaptation to irreversible cellular damage. Antioxidants and Redox Signaling 9 2345-2355.

Hong J, Zhang G, Dong F \& Rechler MM 2002 Insulin-like growth factor (IGF)-binding protein-3 mutants that do not bind IGF-I or IGF-II stimulate apoptosis in human prostate cancer cells. Journal of Biological Chemistry 277 10489-10497.

Jaques G, Noll K, Wegmann B, Witten S, Kogan E, Radulescu RT \& Havemann K 1997 Nuclear localization of insulin-like growth factor binding protein 3 in a lung cancer cell line. Endocrinology 138 1767-1770.

Jia Y, Lee KW, Swerdloff RS, Hwang D, Cobb LJ, Sinha Hikim A, Lue YH, Cohen P \& Wang C 2009 The interaction of insulin-like growth factor binding protein-3 and bax in mitochondria promotes male germ cell apoptosis. Journal of Biological Chemistry 285 1726-1732.

Kim FJ, Beeche AA, Hunter JJ, Chin DJ \& Hope TJ 1996 Characterization of the nuclear export signal of human T-cell lymphotropic virus type 1 Rex reveals that nuclear export is mediated by position-variable hydrophobic interactions. Molecular and Cellular Biology 16 5147-5155.

Lalou C, Lassarre C \& Binoux M 1996 A proteolytic fragment of insulin-like growth factor (IGF) binding protein-3 that fails to bind IGFs inhibits the mitogenic effects of IGF-I and insulin. Endocrinology 137 3206-3212.
Lee KW, Liu B, Ma L, Li H, Bang P, Koeffler HP \& Cohen P 2004 Cellular internalization of insulin-like growth factor binding protein-3: distinct endocytic pathways facilitate re-uptake and nuclear localization. Journal of Biological Chemistry 279 469-476.

Lee KW, Ma L, Yan X, Liu B, Zhang XK \& Cohen P 2005 Rapid apoptosis induction by IGFBP-3 involves an insulin-like growth factor-independent nucleomitochondrial translocation of RXRalpha/Nur77. Journal of Biological Chemistry 280 16942-16948.

Lee KW, Cobb LJ, Paharkova-Vatchkova V, Liu B, Milbrandt J \& Cohen P 2007 Contribution of the orphan nuclear receptor Nur77 to the apoptotic action of IGFBP-3. Carcinogenesis 28 1653-1658.

Leu JI \& George DL 2007 Hepatic IGFBP1 is a prosurvival factor that binds to BAK, protects the liver from apoptosis, and antagonizes the proapoptotic actions of p53 at mitochondria. Genes and Development 21 3095-3109.

Li W, Fawcett J, Widmer HR, Fielder PJ, Rabkin R \& Keller GA 1997 Nuclear transport of insulin-like growth factor-I and insulin-like growth factor binding protein-3 in opossum kidney cells. Endocrinology 138 1763-1766.

Lithgow T, van Driel R, Bertram JF \& Strasser A 1994 The protein product of the oncogene bcl-2 is a component of the nuclear envelope, the endoplasmic reticulum, and the outer mitochondrial membrane. Cell Growth and Differentiation 5 411-417.

Liu B, Lee HY, Weinzimer SA, Powell DR, Clifford JL, Kurie JM \& Cohen P 2000 Direct functional interactions between insulin-like growth factor- binding protein-3 and retinoid $X$ receptor-alpha regulate transcriptional signaling and apoptosis. Journal of Biological Chemistry 275 33607-33613.

Liu B, Lee KW, Anzo M, Zhang B, Zi X, Tao Y, Shiry L, Pollak M, Lin S \& Cohen P 2007 Insulin-like growth factor-binding protein-3 inhibition of prostate cancer growth involves suppression of angiogenesis. Oncogene 26 1811-1819.

Malhotra JD \& Kaufman RJ 2007 Endoplasmic reticulum stress and oxidative stress: a vicious cycle or a doubleedged sword? Antioxidants and Redox Signaling 9 2277-2293.

Mihara M, Erster S, Zaika A, Petrenko O, Chittenden T, Pancoska P \& Moll UM 2003 p53 has a direct apoptogenic role at the mitochondria. Molecular Cell 11 577-590.

Mishra S, Raz A \& Murphy LJ 2004 Insulin-like growth factor binding protein-3 interacts with autocrine motility factor/phosphoglucose isomerase (AMF/PGI) and inhibits the AMF/PGI function. Cancer Research 64 2516-2522.

Moll UM, Marchenko N \& Zhang XK 2006 p53 and Nur77/TR3 - transcription factors that directly target mitochondria for cell death induction. Oncogene $\mathbf{2 5}$ 4725-4743. 
Radulescu RT 1994 Nuclear localization signal in insulinlike growth factor-binding protein type 3. Trends in Biochemical Science 19278.

Rajah R, Valentinis B \& Cohen P 1997 Insulin-like growth factor (IGF)-binding protein-3 induces apoptosis and mediates the effects of transforming growth factor-beta1 on programmed cell death through a p53- and IGFindependent mechanism. Journal of Biological Chemistry 272 12181-12188.

Ricort JM, Lombet A, Lassarre C \& Binoux M 2002 Insulinlike growth factor binding protein-3 increases intracellular calcium concentrations in MCF-7 breast carcinoma cells. FEBS Letters 527 293-297.

Rizzuto R, Pinton P, Carrington W, Fay FS, Fogarty KE, Lifshitz LM, Tuft RA \& Pozzan T 1998 Close contacts with the endoplasmic reticulum as determinants of mitochondrial $\mathrm{Ca}^{2+}$ responses. Science 280 1763-1766.

Santer FR, Bacher N, Moser B, Morandell D, Ressler S, Firth SM, Spoden GA, Sergi C, Baxter RC, Jansen-Durr P et al. 2006 Nuclear insulin-like growth factor binding protein-3 induces apoptosis and is targeted to ubiquitin/proteasomedependent proteolysis. Cancer Research 66 3024-3033.

Schedlich LJ, Young TF, Firth SM \& Baxter RC 1998 Insulin-like growth factor-binding protein (IGFBP)-3 and IGFBP-5 share a common nuclear transport pathway in T47D human breast carcinoma cells. Journal of Biological Chemistry 273 18347-18352.

Schedlich LJ, Le Page SL, Firth SM, Briggs LJ, Jans DA \& Baxter RC 2000 Nuclear import of insulin-like growth factor-binding protein- 3 and -5 is mediated by the importin beta subunit. Journal of Biological Chemistry 275 23462-23470.

Schedlich LJ, O'Han MK, Leong GM \& Baxter RC 2004 Insulin-like growth factor binding protein-3 prevents retinoid receptor heterodimerization: implications for retinoic acid-sensitivity in human breast cancer cells. Biochemical and Biophysical Research Communications 314 83-88.

Schedlich LJ, Muthukaruppan A, O'Han MK \& Baxter RC 2007 Insulin-like growth factor binding protein-5 interacts with the vitamin $\mathrm{D}$ receptor and modulates the vitamin D response in osteoblasts. Molecular Endocrinology 21 2378-2390.

Scorrano L, Oakes SA, Opferman JT, Cheng EH, Sorcinelli MD, Pozzan T \& Korsmeyer SJ 2003 BAX and BAK regulation of endoplasmic reticulum $\mathrm{Ca}^{2+}$ : a control point for apoptosis. Science 300 135-139.

Sell C, Rubini M, Rubin R, Liu JP, Efstratiadis A \& Baserga R 1993 Simian virus 40 large tumor antigen is unable to transform mouse embryonic fibroblasts lacking type 1 insulin-like growth factor receptor. PNAS $\mathbf{9 0}$ 11217-11221.

Silha JV, Sheppard PC, Mishra S, Gui Y, Schwartz J, Dodd JG \& Murphy LJ 2006 Insulin-like growth factor (IGF) binding protein-3 attenuates prostate tumor growth by IGF-dependent and IGF-independent mechanisms. Endocrinology 147 2112-2121.

Spagnoli A, Torello M, Nagalla SR, Horton WA, Pattee P, Hwa V, Chiarelli F, Roberts CT Jr \& Rosenfeld RG 2002 Identification of STAT-1 as a molecular target of IGFBP-3 in the process of chondrogenesis. Journal of Biological Chemistry 277 18860-18867.

Stommel JM, Marchenko ND, Jimenez GS, Moll UM, Hope TJ \& Wahl GM 1999 A leucine-rich nuclear export signal in the p53 tetramerization domain: regulation of subcellular localization and p53 activity by NES masking. EMBO Journal 18 1660-1672.

Stone SJ \& Vance JE 2000 Phosphatidylserine synthase-1 and -2 are localized to mitochondria-associated membranes. Journal of Biological Chemistry 275 34534-34540.

Sun T, Hunziker EB \& Morales TI 2008 Subcellular distribution of the insulin-like growth factor (IGF) binding proteins (IGFBPs) 2 and 3 in articular chondrocytes. Journal of Orthopaedic Research 26 1421-1427.

Valentinis B, Bhala A, DeAngelis T, Baserga R \& Cohen P 1995 The human insulin-like growth factor (IGF) binding protein-3 inhibits the growth of fibroblasts with a targeted disruption of the IGF-I receptor gene. Molecular Endocrinology 9 361-367.

Vance JE 1990 Phospholipid synthesis in a membrane fraction associated with mitochondria. Journal of Biological Chemistry 265 7248-7256.

Wraight CJ, Liepe IJ, White PJ, Hibbs AR \& Werther GA 1998 Intranuclear localization of insulin-like growth factor binding protein- 3 (IGFBP-3) during cell division in human keratinocytes. Journal of Investigative Dermatology 111 239-242.

Yamamoto H \& Murphy LJ 1994 Generation of des-(1-3) insulin-like growth factor-I in serum by an acid protease. Endocrinology 135 2432-2439.

Yamamoto H \& Murphy LJ 1995 Enzymatic conversion of IGF-I to des(1-3) IGF-I in rat serum and tissues: a further potential site of growth hormone regulation of IGF-I action. Journal of Endocrinology 146 141-148.

Yamanaka Y, Fowlkes JL, Wilson EM, Rosenfeld RG \& Oh Y 1999 Characterization of insulin-like growth factor binding protein-3 (IGFBP-3) binding to human breast cancer cells: kinetics of IGFBP-3 binding and identification of receptor binding domain on the IGFBP-3 molecule. Endocrinology 140 1319-1328.

Yan X, Forbes BE, McNeil KA, Baxter RC \& Firth SM 2004 Role of N- and C-terminal residues of insulinlike growth factor (IGF)-binding protein-3 in regulating IGF complex formation and receptor activation. Journal of Biological Chemistry 279 53232-53240. 